\section{Choosing Wisely: Highest-Cost Tests in Outpatient Neurology}

\author{
James F. Burke MD, MS, ${ }^{1,2}$ \\ Lesli E. Skolarus MD, MS, ${ }^{2}$ \\ Brian C. Callaghan MD, $\mathrm{MS}^{3}$ and \\ Kevin A. Kerber MD, MS $^{3}$
}

Identifying the tests/procedures ordered by neurologists that contribute most to health care expenditures is a critical step in the process of creating the neurology top 5 list for the Choosing Wisely initiative. Using data from the 2007-2010 National Ambulatory Care Medical Survey, we found that $\$ 13.3$ billion $(95 \%$ confidence inter$\mathrm{val}=\$ 10.1-\$ 16.5$ billion) was spent on tests ordered at neurologist visits. The tests/procedures with the highest expenditures were magnetic resonance imaging (MRI; $51 \%$ of total expenditures; $\$ 7.5$ billion), electromyography (EMG; $20 \%$ of expenditures; $\$ 2.6$ billion), and electroencephalography (EEG; $8 \%$ of expenditures; $\$ 1.1$ billion). MRI, EMG, and EEG should receive close scrutiny in the development of the neurology top 5 list.

ANN NEUROL 2013;73:679-683

In response to unsustainable growth in health care expenditures, ${ }^{1}$ the American Board of Internal Medicine launched the Choosing Wisely initiative. ${ }^{2,3}$ As part of this initiative, medical professional societies have been encouraged to identify 5 non-value-added tests or procedures commonly used in their field, whose necessity should be carefully scrutinized. One potential virtue of this approach is that by identifying and decreasing the use of low-value tests/procedures, physicians demonstrate to a skeptical public that they are genuinely protecting patients' interests rather than rationing health care. ${ }^{3}$

So far, 9 specialty societies have each developed a top 5 list. Examples of list items include: "Don't obtain imaging studies in patients with non-specific low back pain" and "In the evaluation of simple syncope and a normal neurological examination, don't obtain brain imaging studies (CT or MRI)" (American College of Physicians). ${ }^{4}$ The American Academy of Neurology recently joined the Choosing Wisely initiative and plans to release a top 5 list in $2013 .^{5}$

A recent critique of the first of the published top 5 lists is that many of the tests/procedures had only marginal, or in some cases negligible, impact on health care costs. ${ }^{6,7}$ As a result, it has been recommended that future top 5 list development efforts should incorporate cost information to ensure that high-impact services are addressed. ${ }^{6}$ In this study, we sought to define the tests and procedures associated with the highest expenditures in outpatient neurologic care and identify the clinical scenarios where those tests are most commonly used.

\section{Patients and Methods}

\section{Data Set}

The National Ambulatory Medical Care Survey (NAMCS) is a nationally representative survey conducted annually by the Centers for Disease Control and Prevention. ${ }^{8}$ NAMCS is designed using a 3-stage sampling design (geographic regions, physician practices stratified within specialties, and patient visits within practices) to enable a nationally representative characterization of outpatient office-based care. For this study, we analyzed all neurologist visits in NAMCS from 2007 to 2010. This sample includes data from 125,029 visits, including 6,764 visits by 195 unique neurologists.

\section{Diagnoses}

The principal NAMCS diagnosis for each visit (using International Classification of Diseases, 9th Edition, Clinical Modification [ICD-9-CM] codes) was used to categorize diagnoses with Healthcare Cost and Utilization Project multilevel diagnosis Clinical Classifications Software. ${ }^{9}$ For these analyses, each principal diagnosis was categorized by the lowest level diagnostic category in which it was classified.

\section{Tests/Procedures}

Test/procedure utilization data are abstracted onto the standardized NAMCS survey instrument by provider practices either by checking boxes for specific procedures (magnetic resonance imaging $[\mathrm{MRI}]$, computed tomography $[\mathrm{CT}]$, $\mathrm{x}$-rays, some laboratory tests, and ultrasound studies) or by handwriting test/procedure names in available additional space if no checkbox is available (eg, electromyography [EMG], electroencephalography [EEG], polysomnography [PSG]). Handwritten tests/ procedures are later translated into ICD-9 procedure codes.

From the ${ }^{1}$ Department of Veterans Affairs, VA Center for Clinical Management and Research, Ann Arbor, VA Healthcare System; ${ }^{2}$ Stroke Program, University of Michigan; University of Michigan, Ann Arbor, Ml; ${ }^{3}$ Department of Neurology, University of Michigan, Ann Arbor, MI.

Address correspondence to Dr Burke, RWJ Clinical Scholars Program, North Campus Research Complex, 2800 Plymouth Rd, Building \#10, Room G016, Ann Arbor, MI 48109. E-mail: jamesbur@umich.edu

Additional Supporting Information may be found in the online version of this article.

View this article online at wileyonlinelibrary.com. DOI: 10.1002/ ana.23865

Received Nov 21, 2012, and in revised form Jan 9, 2013. Accepted for publication Feb 1, 2013. 
TABLE 1. All Principal Diagnoses for Neurologist Visits with $>500,000$ Visits (Classified by Most Distal Multilevel CCS Category) in National Ambulatory Medical Care Survey from 2007 to 2010

\begin{tabular}{|c|c|c|}
\hline Diagnosis & $\begin{array}{l}\text { Total No. of Visits, } \\
\text { in Millions }(95 \% \mathrm{CI})\end{array}$ & $\begin{array}{l}\text { Average No. of } \\
\text { Visits per Year, } \\
\text { in Millions }(95 \% \mathrm{CI})\end{array}$ \\
\hline Disorders of the peripheral nervous system & $6.03(4.32-7.74)$ & $1.51(1.08-1.93)$ \\
\hline Migraine & $5.01(3.55-6.47)$ & $1.25(0.89-1.62)$ \\
\hline Other back problems & $4.00(2.42-5.57)$ & $1.00(0.61-1.39)$ \\
\hline Epilepsy & $3.92(2.65-5.19)$ & $0.98(0.66-1.30)$ \\
\hline Residual codes, unclassified $^{a}$ & $3.01(1.55-4.46)$ & $0.75(0.39-1.12)$ \\
\hline Other headache & $2.62(1.81-3.44)$ & $0.66(0.45-0.86)$ \\
\hline Convulsions & $2.56(1.77-3.35)$ & $0.64(0.44-0.84)$ \\
\hline Other nervous system symptoms and disorders ${ }^{b}$ & $2.52(1.73-3.31)$ & $0.63(0.43-0.83)$ \\
\hline Parkinson disease & $2.5(1.75-3.25)$ & $0.62(0.44-0.81)$ \\
\hline $\begin{array}{l}\text { Delirium, dementia, amnestic, and other } \\
\text { cognitive disorders }\end{array}$ & $2.29(1.59-3)$ & $0.57(0.40-0.75)$ \\
\hline Multiple sclerosis & $2.25(1.34-3.17)$ & $0.56(0.33-0.79)$ \\
\hline $\begin{array}{l}\text { Other hereditary and degenerative } \\
\text { nervous system conditions }\end{array}$ & $1.85(1.35-2.35)$ & $0.46(0.34-0.59)$ \\
\hline Other connective tissue disease & $1.84(1.17-2.52)$ & $0.46(0.29-0.63)$ \\
\hline Acute cerebrovascular disease & $1.3(0.83-1.77)$ & $0.33(0.21-0.44)$ \\
\hline Other central nervous system disorders & $1.29(0.78-1.8)$ & $0.32(0.16-0.45)$ \\
\hline Intervertebral disk disorders & $1.19(0.34-2.03)$ & $0.30(0.09-0.51)$ \\
\hline Conditions associated with dizziness or vertigo & $1.03(0.71-1.35)$ & $0.26(0.18-0.34)$ \\
\hline Missing & $0.72(0.37-1.08)$ & $0.18(0.09-0.51)$ \\
\hline Transient cerebral ischemia & $0.67(0.32-1.01)$ & $0.17(0.08-0.25)$ \\
\hline $\begin{array}{l}\text { Attention deficit disorder and attention deficit } \\
\text { hyperactivity disorder }\end{array}$ & $0.62(0.15-1.1)$ & $0.16(0.04-0.27)$ \\
\hline Other aftercare ${ }^{c}$ & $0.56(0.18-0.95)$ & $0.14(0.05-0.24)$ \\
\hline Syncope & $0.55(0.32-0.78)$ & $0.14(0.08-0.20)$ \\
\hline Spondylosis and allied disorders & $0.55(0.2-0.89)$ & $0.14(0.05-0.22)$ \\
\hline
\end{tabular}

Tests/procedures were included in this study if they appeared in the sample 5 or more times. Laboratory tests were combined into an index variable representing the total number of laboratory tests used in a given patient.

\section{Payments}

Payments were determined using the Medicare physician fee schedule and Medicare Clinical Laboratory Fee Schedule for tests/procedures and laboratory tests, respectively. ${ }^{10,11}$ Total payments were calculated by adding all payments for all tests/procedures. The NAMCS survey instrument does not capture what portion of the body was imaged for CT or MRI, and ICD-9CM procedure codes for EMG and EEG do not offer sufficient detail to determine the average payments associated with these procedures. Consequently, we used all Medicare claims associated with the Health and Retirement Study (HRS) population, ${ }^{12}$ a nationally representative sample, to estimate the payments associated with the average test (eg, the average MRI or the average EMG) ordered by a neurologist. To estimate this average for each test, we determined the distribution of test components performed when ordered by a neurologist. In this way, we were able to account for variation in number of body 
segments tested and protocols. After determining the distribution of test components per average test, we applied the national limit payment amounts for each test component separately and summed these components to determine the payment for an average test. For comparison purposes, the total expenditures on evaluation and management (E\&M) services were estimated using a similar approach. The proportion of individual E\&M codes used by neurologists was calculated in the Medicare HRS population, and then the cost of the average neurologist visit was estimated by taking a weighted average of national limit payment amounts.

\section{Statistical Analysis}

Descriptive statistics using survey weights were used to estimate the number of visits for each diagnosis category, total expenditures for all test categories, and expenditures for all tests by diagnostic categories. All analyses were performed in Stata version 12.1 (StataCorp, College Station, TX).

\section{Results}

A total of 58 million $(95 \%$ confidence interval $[\mathrm{CI}]=44-72$ million) visits to the neurologist were identified from 2007 to 2010. The 3 most common diagnostic categories were disorders of the peripheral nervous system, migraine, and back pain (Table 1).

From 2007 to 2010, a total of $\$ 13.3$ billion (95\% $\mathrm{CI}=\$ 10.1-\$ 16.5$ billion) was spent on diagnostic tests ordered at neurologist visits, with a minimum of $\$ 3.2$ billion spent in every year (Table 2). MRI accounted for $57 \%$ of all diagnostic expenditures ( $\$ 7.5$ billion; $95 \%$ $\mathrm{CI}=\$ 5.7-\$ 9.4$ billion). EMG accounted for $20 \%$ of expenditures ( $\$ 2.6$ billion; 95\% CI $=\$ 1.9-\$ 3.3$ billion) and EEG for $8.3 \%$ ( $\$ 1.1$ billion; $95 \% \mathrm{CI}=\$ 0.7-\$ 1.5$ billion). Together, EMG and EEG accounted for $64 \%$ of the non-MRI-related expenditures. Expenditures associated with PSG increased over time, but were $<5 \%$ of total expenditures in 2010. Laboratory testing accounted for only $1.6 \%$ of overall expenditures. By comparison, a total of $\$ 6.1$ billion (95\% CI $=\$ 4.7-\$ 7.6$ billion) was spent on all evaluation and management services attributable to neurologists over this time period.

The diagnostic category with the highest single test expenditures was disorders of the peripheral nervous system, with EMG costs of $\$ 820$ million $(95 \%$ $\mathrm{CI}=\$ 520$ million- $\$ 1.1$ billion; Fig). The only other diagnostic category with EMG expenditures $>\$ 250$ million was "other back problems." The diagnostic category of migraine had the second highest single-test expenditures, with MRI costs of $\$ 690$ million $(95 \% \mathrm{CI}=\$ 350$ million-\$1 billion). In contrast with EMG, MRI had 12 diagnostic categories with expenditures of $\$ 250$ million or more. For EEG, epilepsy was the only diagnostic category with expenditures totaling $>\$ 200$ million (Supplementary Table).

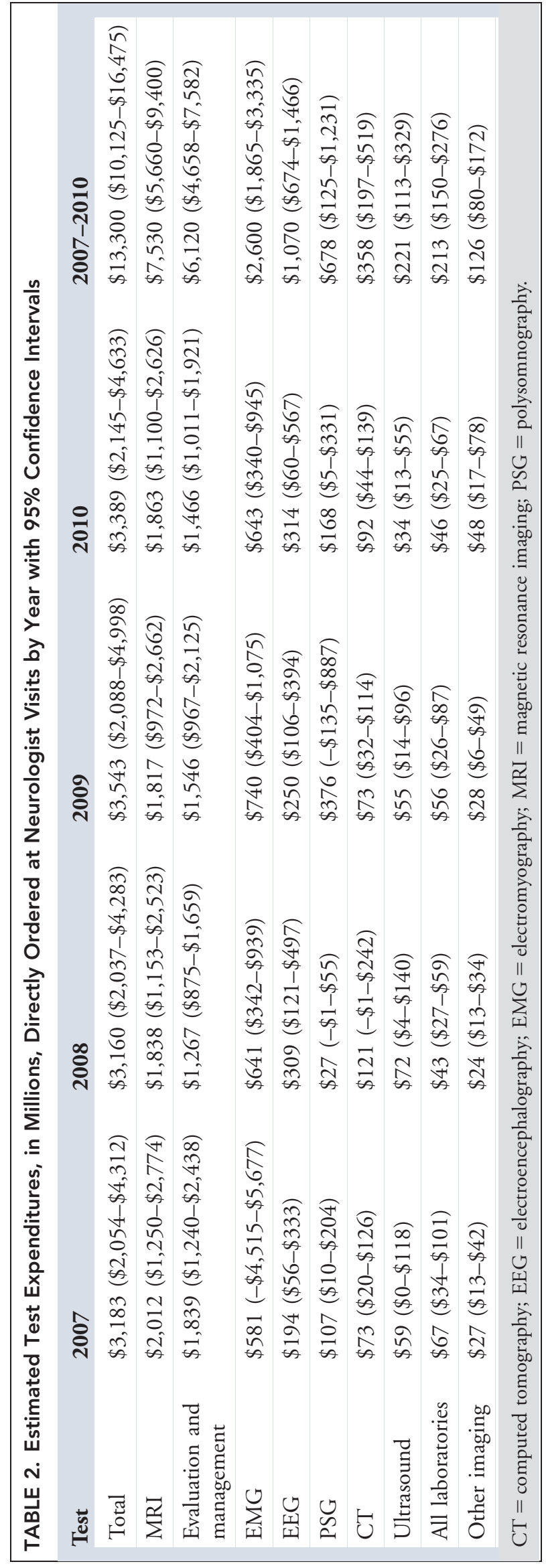




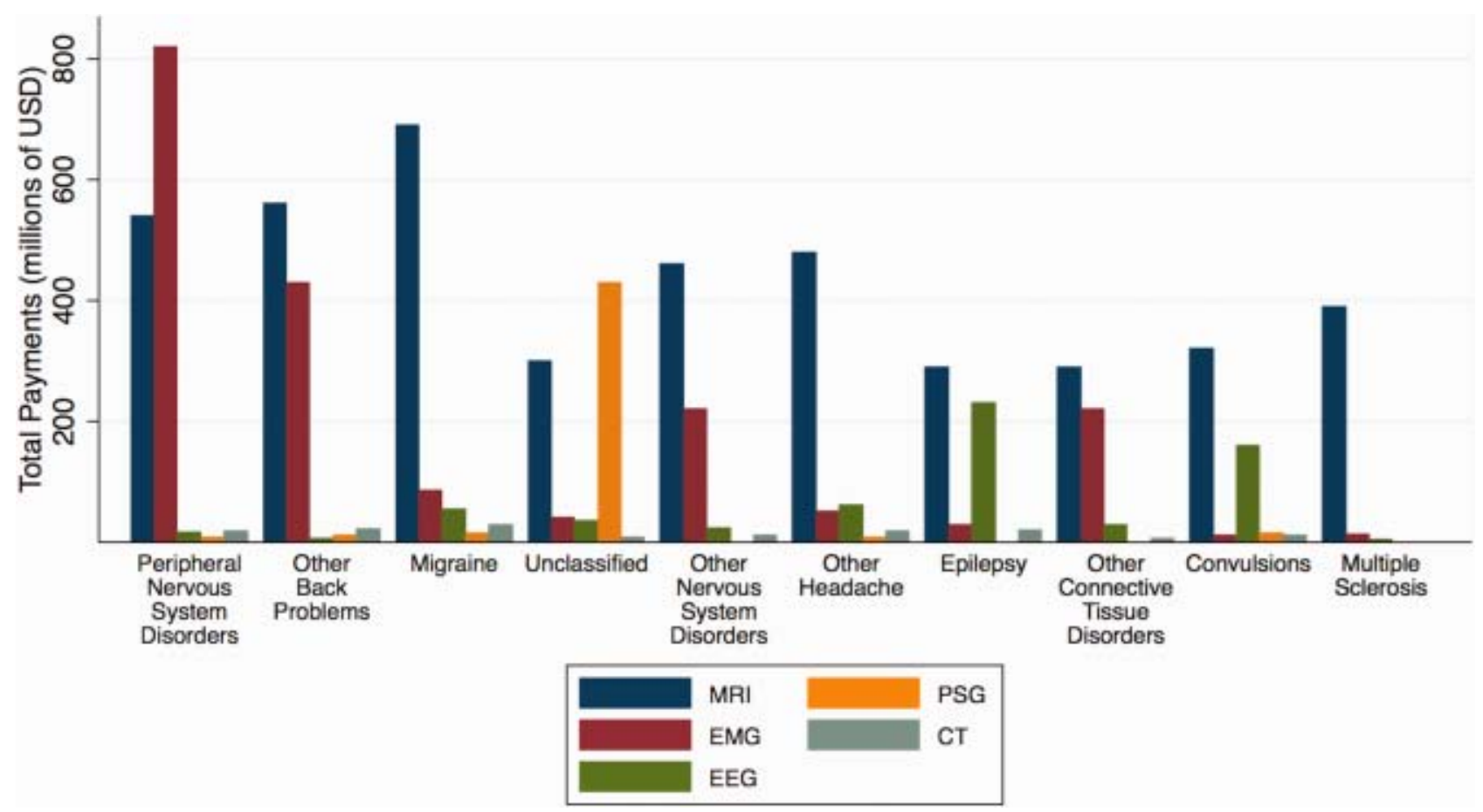

FIGURE : Estimated payments for the 5 most costly tests for the 10 most common diagnostic categories. CT $=$ computed tomography; $E E G$ = electroencephalography; $E M G=$ electromyography; $M R I=$ magnetic resonance imaging; $P S G=$ polysomnography; USD = US dollars. [Color figure can be viewed in the online issue, which is available at www.annalsofneurology.org.]

\section{Discussion}

The Choosing Wisely campaign aims to reduce unsustainable health care expenditures by identifying clinical contexts in which specific tests/procedures may be wasteful. Identifying targets in neurology requires consideration first of the net clinical utility of a given test/procedure and secondarily of financial costs. In this study, we identified the highest-cost tests/procedures in outpatient care ordered by neurologists. Given their contributions to overall expenditures, MRI, EMG, and EEG should receive close scrutiny in the development of the neurology top 5 list, as together these tests account for $84 \%$ of all costs attributable to outpatient neurologist care.

Due to their relatively low expenditures, laboratory tests, CT, ultrasound, and other imaging studies should be given a lower priority in the development of the neurology top 5 list. PSG expenditures may increase in importance if the observed increases from 2007 to 2010 continue into the future.

The primary remaining challenge in developing a top 5 list is to identify the specific clinical scenarios where these tests are of sufficiently low value as to be considered unnecessary or even wasteful. ${ }^{4}$ Items in top 5 lists are typically written to discourage the use of a specific test within a specific clinical circumstance. ${ }^{4}$ We found that the expenditures for MRI were widely dispersed among 12 different diagnostic categories. EMG and EEG expenditures were more concentrated within the diagnostic categories, although those specific categories (eg, disorders of the peripheral nervous system, epilepsy) are sufficiently broad to necessitate additional steps to identify specific clinical scenarios in which these expensive tests do not add value. Given the paucity of data on the net clinical value of tests/procedures, research studies designed to define the value of these tests in specific clinical scenarios are needed to ensure that waste reduction efforts can be based on evidence.

Other strategies are being used to address test/procedure-related expenditures such as recently implemented coding and payment changes for EMG/nerve conduction studies. The impact of such across-the-board cuts on expenditures remains to be determined. Given the magnitude of expenditure difference between MRI, EMG, EEG, and other tests, however, the relative expenditure rankings of these tests is unlikely to change unless there is a substantial decline in expenditures for 1 test. If such broad reimbursement changes do reduce overall expenditures, they may not do so in a way that targets actual waste while preserving value, which is the aim of more focused expenditure-reduction efforts, such as the Choosing Wisely campaign. ${ }^{13,14}$

This study is limited by the available data from the NAMCS data set and the estimates of payments available. These data include little detail on the specifics of tests that were ordered, and thus we standardized costs to the cost of an average test (eg, the average MRI or average EMG 
ordered by neurologists). This approach should lead to unbiased and conservative (by failing to account for higher payments paid by private insurers) estimates of overall payments, but may introduce some bias for specific clinical scenarios where a more or less expensive version of a test is used compared to the average. Finally, NAMCS offers limited clinical detail on the circumstances surrounding why specific tests were ordered, limiting inferences about the value of the tests/procedures identified here. Without such details, we cannot conclude that any test or procedure is overused; nor can we exclude the possibility of underutilization in appropriate clinical contexts. Rather, these data simply establish which tests/procedures account for the most resources.

\section{Acknowledgment}

This work was supported by NINDS K23 NS073685 (L.E.S.) AHRQ R18 HS017690 (K.A.K), and an American Diabetes Association Junior Faculty Award (B.C.C.). Travel support was provided by the Ann Arbor Veterans Administration (J.F.B.).

\section{Potential Conflicts of Interest}

L.E.S.: grants/grants pending, NIH; travel expenses, ANA/NINDS. B.C.C.: grants/grants pending, NIH K23 Award.

\section{References}

1. Berwick DM, Hackbarth AD. Eliminating waste in US health care. JAMA 2012;307:1513-1516.
2. Choosing Wisely: about the campaign. Available at: http:// choosingwisely.org/wp-content/uploads/2012/11/choosing-wiselyone-pager.pdf Accessed November 13, 2012.

3. Brody H. Medicine's ethical responsibility for health care reformthe Top Five list. N Engl J Med 2010;362:283-285

4. Choosing Wisely: five things physicians and patients should question. Available at: http://choosingwisely.org/wp-content/uploads/ 2012/04/Five-Things.pdf Accessed November 13, 2012.

5. American Board of Internal Medicine. Choosing Wisely. Available at: http://www.abimfoundation.org/Initiatives/Choosing-Wisely. aspx Accessed November 13, 2012.

6. Kale MS, Bishop TF, Federman AD, Keyhani S. "Top 5" lists top $\$ 5$ billion. Arch Intern Med 2011;171:1856-1858.

7. The "top 5" lists in primary care: meeting the responsibility of professionalism. Arch Intern Med 2011;171:1385-1390.

8. National Center for Health Statistics. 2007-2010 National Ambulatory Medical Care Survey (NAMCS), public use data files. Available at: ftp://ftp.cdc.gov/pub/Health_Statistics/NCHS/Datasets/NAMCS/. Accessed January 2, 2013.

9. Agency for Healthcare Research and Quality. Clinical Classifications Software (CCS) for ICD-9-CM fact sheet. Available at: http:// www.hcup-us.ahrq.gov/toolssoftware/ccs/ccsfactsheet.jsp Accessed November 19, 2012.

10. Centers for Medicare \& Medicaid Services. Physician fee schedule search. Available at: http://www.cms.gov/apps/physician-feeschedule/search/search-criteria.aspx. Accessed February 15, 2013.

11. Centers for Medicare \& Medicaid Services. Clinical laboratory fee schedule. Available at: http://www.cms.gov/Medicare/MedicareFee-for-Service-Payment/ClinicalLabFeeSched. Accessed September 1, 2012.

12. Health and retirement study. Ann Arbor, MI: University of Michigan, 2007.

13. American Association of Neuromuscular \& Electrodiagnostic Medicine. 2013 coding changes significant reduce EDX RVUs. Available at: http://www.aanem.org/About-Us/News/2013-Coding-ChangesSignificantly-Reduce-EDX-RVUs.aspx Accessed January 2, 2013.

14. American Academy of Neurology. NCS cuts go into effect January 1. Available at: http://www.aan.com/news/?event=read\&article id=10982 Accessed 1/2/2013. 